\title{
Cross-Cultural Multimedia Language Learning: Case Study and Analysis
}

\author{
Carsten Ullrich ${ }^{1}$, Kerstin Borau ${ }^{2}$, Scott Grant ${ }^{3}$, Daniel Jackson ${ }^{4}$, Ruimin Shen ${ }^{1}$ \\ ${ }^{1}$ Shanghai Jiao Tong University, 1954 Hua Shan Road, 200050 Shanghai, China \\ ${ }^{2}$ Distance Education College of SJTU, 500 Jiangsu Road, 200050 Shanghai, China \\ ${ }^{3}$ Faculty of Arts, Monash University, Wellington Road, Clayton, 3800, Australia \\ ${ }^{4}$ Manifest and Process LLC, Boulder, Colorado, USA \\ ullrich_c@sjtu.edu.cn, kerstin.borau@gmail.com,scott.grant@arts.monash.edu.au,rab- \\ bidanielhjackson@gmail.com,rmshen@sjtu.edu.cn
}

\begin{abstract}
This paper describes a pedagogical pattern for cross-cultural language learning and its application in a case study involving learners in Australia and China. The pattern uses a multimedia discussion tool as its main component, supported by video conferencing and chat. We analyze how students interacted with the tools, especially the documents they created and the commenting behavior. Based on this analysis, we give a set of recommendations relevant to practitioners and researchers in technology-enhanced language learning.
\end{abstract}

Keywords: CALL, EFL, multimedia, cross-cultural, Web 2.0

\section{Introduction}

Today's views on language learning emphasize communication, interaction and authenticity. Learning communicative competence [10] and interaction has become an integral part of communicative language learning [4]; language as discourse implies using real, complete and authentic situations [5]. The Internet facilitates communication, interaction and cross-cultural learning tremendously. The following paper describes how several Internet-based tools were used in blended classrooms to promote the production of spoken language using synchronous and asynchronous tools for collaborative learning tasks and intercultural exchange.

\section{Description of the Participants}

This case-study brought together students from Shanghai, China and Monash, Australia. The 82 Chinese students were adult learners (ages 20-30) enrolled at Shanghai Jiao Tong's Distance Education College, which offers blended classrooms that allow students to participate in "real" classrooms and by watching the classes at home, live and recorded. The students enrolled in the unit "Newspaper Discussion" that focused on current news. The course took place 3 hours once a week for 15 weeks. The 31 Australian students are undergraduates (ages 18-22) enrolled in the unit "Chinese 
Media Studies (CMS)". CMS focuses on Chinese domestic and international news and current affairs as seen through the eyes of the online Chinese media. Each week for 13 weeks students attend a 2 hour seminar focused on text-based media and a one hour computer lab focused on Internet based media and tools. Classes are face to face.

\section{A Pedagogical Pattern for Cross-Cultural Language Learning}

We planned the cross-cultural language learning to take place in the following way. A synchronous video conference using Skype serves as the kick-off meeting in which the instructors and students from both classes introduced themselves. At the same time, the joint chat group is created to allow for asynchronous communication. Then, a teacher creates the first multimedia document. For this purpose, we have been using the free Web tool VoiceThread (VT). In our case study, the Australian instructor recorded a video where he introduced himself. In addition, teacher and students jointly create a document. These two activities help the learners to get used to the tool. In the following weeks students upload documents, either created at home or in class. By using an asynchronous tool, students can participate whenever they have time. The teachers encourage commenting on the documents by several classroom activities: commenting from the classroom and by discussing documents and comments.

\section{Analysis}

Student Presentations The students produced a significant amount of multimedia documents, in total 43, with 15 authored by Australian students and 28 authored by Chinese. 5 threads consisted of videos (4 Australian, 1 Chinese), 21 of pictures (1 Australian, 20 Chinese). Students did not interact similarly with all threads. Only slightly more than half of all threads received at least 1 comment (5 Australian threads, 21 Chinese threads). Of the 82 enrolled Chinese students, 17 produced at least $1 \mathrm{VT}$. This number is low but corresponds to the college's average number of students who hand in homework (20-25\%). The Australian students were divided up into groups of 4 or 5 and all 8 groups produced at least one thread.

The threads covered a wide range of topics, with significant difference between the two classes. About $1 / 3$ of the threads (10) created by the Chinese students covered cultural topics, such as differences between Chinese generations, festivals and food. The remaining 2/3 (18) presented aspects of the private life of the students, such as self introductions, job description, holiday pictures, and their home village. This ratio is inversed in the Australian threads. They focused mostly on Australia (11 on tourism, food, animals, educational system, etc). Only 4 threads were of a more personal nature, namely self-introductions and how they spend their time during Easter

The VT titles reflect this difference in personal vs. impersonal threads. Half of the Chinese threads had titles that could be classified as personal, such as "My company life", "My home village". In contrast, Australian titles were more "objective": "Australia's Educational System", "Australian Food". Interestingly, even the threads of a more personal nature carried impersonal titles such as "Easter Australia". 
These different views of the two groups with respect to impersonal and personal topics is also visible in the comment behavior. In total, 26 Chinese students commented, with an average of 6 comments per student. On the Australian side, 19 students commented, with an average of 3 comments per student. Video threads and pictures threads both received a similar amount of comments.

One pattern that is clearly visible from the comment data is that Australian students almost exclusively commented on Chinese threads (3 vs. 63 comments). In contrast, Chinese students commented on Australian and Chinese threads, yet 3 times more often on Chinese threads (37 vs. 121 comments). We believe this data shows different interpretations of the activities. The Australian students were more interested in communicating with their Chinese peers, while the Chinese were more interested in communication in general, regardless whether with their Chinese classmates or the Australian students. In part, these interests may reflect the disciplinary differences between the two groups of students. Students taking courses in a traditional liberal arts program are required to demonstrate objective skills-papers, projects, and scholarship-whereas successful performance in business programs includes interpersonal subjective skills necessary for success in the business world.

Commenting also differed regarding the comment type: the Australian students commented by voice significantly more often than by typed text (59 vs. 7 comments). The Chinese students showed the opposite behavior: 36 students commented by voice, 118 by typed text. The main reason for the frequent usage of voice comments by the Australian students is technical. At the time of usage, VT did not allow typing in Chinese characters. Thus, the only way students could conveniently comment in Chinese was by voice. The Chinese preference for written comment is presumably due to them feeling more secure when writing in contrast to speaking English.

The personal/impersonal distinction visible in the thread topics is also visible in the comments. Chinese students commented on those Australian threads that had a personal focus. The threads about general Australian topics were watched, but almost never commented on.

The commenting behavior on the threads created by the Chinese students shows clear differences between Australian and Chinese students. The thread "Chinese News Report 3: Large Eyes" described the HOPE project, which delivers books to schools in the poorer Western region of China. This thread was one of the two most commented on by the Australian students (17 comments), who showed a high interest in this topic with a social focus. Interestingly, none of the Chinese students commented.

In summary, Chinese students created and commented mostly on threads that covered personal topics, i.e., threads about themselves, while the Australian students created threads about broader more impersonal topics.

\section{Recommendations}

The most significant problem of the multimedia discussion tool was the slow access, a direct consequence from using a third-party service "in the cloud". The advantage is that no installation is required, but this also makes it impossible to install the service on one's own server to lower network latency. Even more severe were the comment- 
ing problems, where a large percentage of comments were not uploaded, which was obviously rather demotivating. One way to circumvent this problem was to record the voice comments, store them as a sound file and then upload the file. However, this requires significant technical competence, which not all students possess.

Recommendations: if possible teachers should preload used materials and not upload live, but previously recorded documents to avoid repeated recording. Developers should allow live creation and also upload of previously authored documents. An input channel that allows contributing with a slow Internet connect is of advantage.

Another major source of problem was identity, rights, and group management. Any system in which users create content needs an easy to use way of organizing contacts and mechanisms notify of new creations. Users should be able to define who has access to authored documents. Furthermore, users need to be able to divide their contacts in groups. VT offers these functionalities, but they are overly confusing. There is no possibility to see at a single glance the documents created by contacts. This resulted in students missing some of their friends' creations. Another source of problems was that students often unintentionally set their threads to private. Making a thread public was difficult and there was no option to set all newly created threads to public.

Recommendations: teachers should create several accounts in class and use these to illustrate how to author threads and notify peers. Yet, students/teachers will still have problems, forget to send out notifications, have incorrect access settings, etc. Developers need to implement easy to use contact and group management, ideally by reusing contact lists from other social networks. Also, users need to be able to modify their privacy and rights management settings in one central place.

Access to statistics is another important feature set, which was also not without problems in our study. While VT provides rudimentary statistics (number of views and of comments), these were unfortunately partly incorrect. As a consequence, any detailed analysis requires manual access to all threads and counting the comments.

Recommendations: as a teacher, check first which data the used tool is collecting, and then decide on a grading scheme based on the available data. Developers should make sure the tool collects and provides data about all actions possible with the tool, on a per user basis (if allowed by the users' privacy settings). For a multimedia discussion tools, this includes creation and modification of a thread, access to it and commenting. The date should be annotated with a time stamp and a user identifier.

The main goal of the cross-cultural language learning class was to stimulate students to actively practice their target language. The used tool offers several ways of producing multimedia content, which we hope would enabled student to participate the way they found most comfortable with. The virtual presence of native speakers aimed at motivating the students by making the authoring tasks less artificial: they produce content that would enable their peers to learn more about the other person, their culture, etc. The analysis shows that the students made use of the different input modalities. They uploaded slide shows, pictures and videos, and also commented by text and voice. Interestingly, restricting the input types can be used as a pedagogical method. In our setting, the Australian students were "forced" to comment by voice because the 
tool did not allow input of Chinese characters. The limited amount of Chinese students who commented by voice was unexpected and might be due to the fact that several students accessed VT from their working place, where voice commenting would be considered obtrusive. In addition, since voice commenting frequently failed due to the slow connection, students presumably abandoned it.

The fact that the authored documents were not only viewed by the teacher but by their own and foreign peers increased the motivation to deliver a good performance. Most of the uploaded threads were original and of high quality. In our experience, when asked to prepare presentations Chinese students often plagiarize by reusing slide set circulating in the Internet. In contrast, in our case study this only happened once.

Recommendations: teachers should make documents as public as possible to increase motivation to deliver high-quality work. Developers should enable multiple ways to show-case created documents, e.g., on the tool homepage, etc.

Communication between both classes did take place, but not to the degree we hoped for. Often, comments containing questions were not answered, possibly due to the thread authors not noticing them. The tool provided for synchronous communication (MSN) was almost never used, because the students were not online at the same time (part time vs. full time students).

Recommendations: teachers need to monitor threads and notify students of unanswered comments. Also, when discussing threads in class, teachers should encourage and upload questions about the presented material. Developers should provide ways to notify authors of comments and also offer incentives for commenting, such as high scores, virtual awards received when having created a specific amount of threads, etc.

\section{Related Work}

Technology-Enhanced Learning, Computer-Mediated Communication (CMC) and Computer-Aided Language Learning are highly active areas of research relevant for the work described in this article. Examples of CMC-based written interaction include cross-cultural learning using chat [4], email [7], blogs [2], audio conferencing [3] and video conferencing $[11,8]$. A common observation in all these approaches is that students are highly favorable of these tools and of the chance of interacting with peers while learning. Also, Levy's analysis highlights the potential of these technologies for culture learning [6]. In parallel to the developments in CMC, creating documents has become easier, first using desktop programs [9], while lately approaches that emphasize the students as producers became popular [1]. For instance, [12] describes a learning system that incorporates several Internet-based teaching activities, including homepage design. Again, students were very much interested in creating personal web pages. However, the web page design was considered being too time consuming. Our work extends prior work by enabling creation, interactivity and contact with native speakers. We explored the usage of a multimedia tool that enables easy creation of slide-shows and videos, and, in contrast to described work, also multimedia commenting, which was extensively used by the students. 


\section{Conclusion}

In sum, we can recommend the application of the described pattern for cross-cultural language learning and we consider our case study a success. The students created a significant amount of original, high quality documents and commented on each other's threads. While doing this, they communicated in the target language and thus had practice opportunities not available otherwise. The students liked being able to communicate, create and share. At the same time we feel that we did not exploit the full potential of our pattern. Unanswered questions in the comments reveal that there were still additional opportunities for communication.

Particularly interesting was the different view on the activities. Australian students created threads on topics, with the goal of doing their homework and practicing the target language. The Chinese students also practiced the target language, but were more interested in threads that described their peers and their activities.

Acknowledgments. This research has received funding from FP7/2007-2013 under grant agreement no 231396.

\section{References}

[1]Downes, S.: E-learning 2.0. eLearn 2005(10) (2005)

[2]Ducate, L.C., Lomicka, L.L.: Exploring the blogosphere: Use of web logs in the foreign language classroom. Computer Assisted Language Learning (21), 9-28 (2008)

[3] Hampel, R., Hauck, M.: Towards an effective use of audio conferencing in distance language courses. Language Learning \& Technology 8(1), 66-82 (2004)

[4]Kitade, K.: L2 learners discourse and SLA theories in CMC: Collaborative interaction in internet chat. Computer Assisted Language Learning 13(2), 143-166 (2000)

[5]Levy, M.: Theory and design in a multimedia CALL project in cross-cultural pragmatics. Computer Assisted Language Learning 12(1), 29-57 (1998)

[6]Levy, M.: Culture, culture learning and new technologies: towards a pedagogical framework. Language Learning \& Technology 11(2), 104-127 (2007)

[7]Liaw, M.L., Johnson, R.J.: E-mail writing as a cross-cultural learning experience. System 29(2), 235-251 (2001)

[8] Nishihori, Y., Nagaoka, K., Nishinaga, N., Tanaka, K., Yamamoto, Y., Sato, H., Harada, M., Shen, R., Feng, J., Kang, M.J.: Enabling a multilateral distance class between china, korea and japan: Effective utilization of networking technologies. In: Mizoguchi, R., Dillenbourg, P., Zhu, Z. (eds.) Proceedings of ICCE. Frontiers in Artificial Intelligence and Applications, vol. 151, pp. 383-386. IOS Press (2006)

[9]Parks, S., Huot, D., Hamers, J., H.-Lemonnier, F.: Crossing boundaries: multimedia technology and pedagogical innovation in a high school class. Language Learning \& Technology 7(1), 28-45 (2003)

[10] Savignon, S.J.: Communicative Competence: Theory and Classroom Practice. McGraw-Hill Humanities/Social Sciences/Languages (1997)

[11] Wang, Y.: Supporting synchronous distance language learning with desktop videoconferencing. Language Learning \& Technology 8, 90-121 (2004)

[12] Yang, S.C., Chen, Y.J.: Technology-enhanced language learning: A case study. Computers in Human Behavior 23(1), 860-879 (2007) 